\title{
Solving Partial Differential Equation with Space- and Time-Fractional Derivatives via Homotopy Decomposition Method
}

\author{
Abdon Atangana $^{1}$ and Samir Brahim Belhaouari ${ }^{2}$ \\ ${ }^{1}$ Institute for Groundwater Studies, Faculty of Natural and Agricultural Sciences, University of the Free State, \\ Bloemfontein 9300, South Africa \\ ${ }^{2}$ Department of Mathematics and Computer Science, College of Science, Alfaisal University, P.O. Box 50927, Riyadh 11533, Saudi Arabia
}

Correspondence should be addressed to Abdon Atangana; abdonatangana@yahoo.fr

Received 17 September 2013; Accepted 9 October 2013

Academic Editor: Muhammet Kurulay

Copyright (C) 2013 A. Atangana and S. B. Belhaouari. This is an open access article distributed under the Creative Commons Attribution License, which permits unrestricted use, distribution, and reproduction in any medium, provided the original work is properly cited.

\begin{abstract}
The analytical solution of the partial differential equation with time- and space-fractional derivatives was derived by means of the homotopy decomposition method (HDM). Some examples are given and comparisons are made. The evaluations show that the homotopy decomposition method is extremely successful and suitable. The achieved results make the steadfastness of the HDM and its wider applicability to fractional differential equation obvious. Additionally, the adding up implicated in HDM is exceptionally undemanding and uncomplicated. It is confirmed that HDM is an influential and professional apparatus for FPDEs. It was also established that HDM is supplementary well organized than the ADM, VIM, HAM, and HPM.
\end{abstract}

\section{Introduction}

Many observable fact in natural science, physics, chemistry, and other knowledge preserve to be illustrated incredibly fruitfully by representations using the supposition of derivatives and integrals with fractional order. Attention in the notion of differentiation and integration to noninteger order has existed since the progress of the conventional calculus [1-3]. By insinuation, mathematical modeling of many physical systems is governed by linear and nonlinear fractional differential equations in various applications in fluid mechanics, viscoelasticity, chemistry, physics, biology, and engineering. Since many fractional differential equations are nonlinear and do not have exact analytical solutions, various numerical and analytic methods have been used to solve these equations [4]. The Adomian decomposition method (ADM) [4], the homotopy perturbation method (HPM) [5], the variational iteration method (VIM) [6], homotopy analysis method $[7,8]$, and other methods have been used to provide analytical approximation to linear and nonlinear problems $[9,10]$. However, the convergence region of the corresponding results is rather small $[9,10]$. One of the popular FDE applications is anomalous diffusion; however, this problem can be effectively solved by radial basis function (RBF) collocation methods [11-13]. In this paper, we use homotopy decomposition method that was recently proposed in [14] to solve partial differential equation that arises in groundwater flow problem. The method was first used to solve time-fractional coupled-korteweg-de-vries equations [15]. The homotopy decomposition method is chosen to solve this nonlinear problem because of the following advantages that the method has over the existing methods. The method does not require the linearization or assumptions of weak nonlinearity [5]. The solutions are not generated in the form of general solution as in the Adomian decomposition method. With ADM, the recursive formula allows repetition of terms in the case of nonhomogeneous partial differential equation, this repetition leads to the noisy solution [16]. The solution obtained is noise-free compared to the variational iteration method [16]. No correctional function is required as in the case of the variational homotopy decomposition method [16]. No Lagrange multiplier is required in the case of the variational iteration method [6]. this method is more 
realistic compared to the method of simplifying the physical problems. If the exact solution of the partial differential equation exists, the approximated solution via the method converges to the exact solution [14]. A construction of a homotopy $v(r, p): \Omega \times[0,1]$ is not needed as in the case of the homotopy perturbation method, because in this case one needs first to continuously deform a difficult problem into another one, which is easy to solve [6]. HDM provides us with a convenient way to control the convergence of approximation series without adapting $h$, as in the case of [17], which is a fundamental qualitative difference in analysis between HDM and other methods.

In this paper, two cases of special interest such as the timefractional foam drainage equation and the space-fractional foam drainage equation are discussed in detail. The paper has been arranged as follows. History of the fractional derivative order is presented in Section 2. In Section 3 the homotopy decomposition method for solving fractional derivative order is described. Complexity of the homotopy decomposition method is discussed in Section 4. In Section 5 the application of HDM to solve the space- and time-fractional partial differential equation is discussed. Conclusions are presented in Section 6.

\section{History of the Fractional Derivative Order}

There is in the literature numereous definitions about fractional derivatives [18-23]. The most popular ones are the Riemann-Liouville and the Caputo derivatives. For Caputo derivative we have

$$
{ }_{0}^{C} D_{x}^{\alpha}(f(x))=\frac{1}{\Gamma(n-\alpha)} \int_{0}^{x}(x-t)^{n-\alpha-1} \frac{d^{n} f(t)}{d t^{n}} d t .
$$

For the case of Riemann-Liouville derivative we have the following definition:

$$
D_{x}^{\alpha}(f(x))=\frac{1}{\Gamma(n-\alpha)} \frac{d^{n}}{d x^{n}} \int_{0}^{x}(x-t)^{n-\alpha-1} f(t) d t
$$

Lately, Jumarie (see $[21,22]$ ) proposed a simple alternative definition to the Riemann-Liouville derivative:

$$
\begin{aligned}
D_{x}^{\alpha}(f(x))= & \frac{1}{\Gamma(n-\alpha)} \frac{d^{n}}{d x^{n}} \\
& \times \int_{0}^{x}(x-t)^{n-\alpha-1}\{f(t)-f(0)\} d t .
\end{aligned}
$$

\section{Basic Idea of the HDM $[14,15]$}

To demonstrate the fundamental design of this technique, we reflect on a universal nonlinear nonhomogeneous fractional partial differential equation with initial conditions of the following structure:

$$
\frac{\partial^{\alpha} G(x, t)}{\partial t^{\alpha}}=L(G(x, t))+N(G(x, t))+l(x, t), \quad \alpha>0,
$$

subject to the initial condition

$$
\begin{aligned}
& D_{0}^{\alpha-k} G(x, 0)=f_{k}(x), \quad(k=0, \ldots, n-1), \\
& D_{0}^{\alpha-n} G(x, 0)=0, \quad n=[\alpha], \\
& D_{0}^{k} G(x, 0)=g_{k}(x), \quad(k=0, \ldots, n-1), \\
& D_{0}^{n} G(x, 0)=0, \quad n=[\alpha],
\end{aligned}
$$

where, $\partial^{\alpha} / \partial t^{\alpha}$ indicates the Caputo or Riemann-Liouville fractional derivative operator, $l$ is a known function, $N$ is the general nonlinear fractional differential operator, and $L$ represents a linear fractional differential operator. The process of the HDM primary pace here is to change the fractional partial differential equation to the fractional partial integral equation by applying the inverse operator $\partial^{\alpha} / \partial t^{\alpha}$ of on both sides of (4) to obtain [15]. In the case of RiemannLiouville fractional derivative [15]

$$
\begin{aligned}
G(x, t)= & \sum_{j=1}^{n-1} \frac{f_{j}(x)}{\Gamma(\alpha-j+1)} t^{\alpha-j}+\frac{1}{\Gamma(\alpha)} \\
& \times \int_{0}^{t}(t-\tau)^{\alpha-1} \\
& \quad \times[L(G(x, \tau))+N(G(x, \tau))+l(x, \tau)] d \tau .
\end{aligned}
$$

In the case of Caputo fractional derivative

$$
\begin{aligned}
G(x, t)= & \sum_{j=1}^{n-1} \frac{g_{j}(x)}{\Gamma(\alpha-j+1)} t^{\alpha-j}+\frac{1}{\Gamma(\alpha)} \\
& \quad \times \int_{0}^{t}(t-\tau)^{\alpha-1} \\
& \quad \times[L(G(x, \tau))+N(G(x, \tau))+l(x, \tau)] d \tau,
\end{aligned}
$$

or in general by putting

$$
\begin{aligned}
& \sum_{j=1}^{n-1} \frac{f_{j}(x)}{\Gamma(\alpha-j+1)} t^{\alpha-j}=l(x, t) \quad \text { or } \\
& f(x, t)=\sum_{j=1}^{n-1} \frac{g_{j}(x)}{\Gamma(\alpha-j+1)} t^{\alpha-j},
\end{aligned}
$$

we obtain

$$
\begin{aligned}
U(x, t)= & T(x, t)+\frac{1}{\Gamma(\alpha)} \\
& \times \int_{0}^{t}(t-\tau)^{\alpha-1} \\
& \quad \times[L(U(x, \tau))+N(U(x, \tau))+f(x, \tau)] d \tau .
\end{aligned}
$$


In the homotopy perturbation method, the fundamental statement is that the solutions can be written as a power series in $p$ :

$$
\begin{gathered}
G(x, t, p)=\sum_{n=0}^{\infty} p^{n} G_{n}(x, t), \\
G(x, t)=\lim _{p \rightarrow 1} G(x, t, p) .
\end{gathered}
$$

The nonlinear term can be decomposed as

$$
N G(x, t)=\sum_{n=0}^{\infty} p^{n} \mathscr{H}_{n}(G),
$$

where $p \in(0,1]$ is an embedding parameter. $\mathscr{H}_{n}(G)$ is the He's polynomials [17] that can be generated by

$$
\begin{array}{r}
\mathscr{H}_{n}\left(G_{0}, \ldots, G_{n}\right)=\frac{1}{n !} \frac{\partial^{n}}{\partial p^{n}}\left[N\left(\sum_{j=0}^{\infty} p^{j} G_{j}(x, t)\right)\right], \\
n=0,1,2 \ldots .
\end{array}
$$

The homotopy decomposition method is obtained by the refined combination of homotopy technique with He's polynomials [15] and is given by

$$
\begin{aligned}
& \sum_{n=0}^{\infty} p^{n} G_{n}(x, t)-T(x, t) \\
&=\frac{p}{\Gamma(\alpha)} \int_{0}^{t}(t-\tau)^{\alpha-1} {\left[l(x, \tau)+L\left(\sum_{n=0}^{\infty} p^{n} G_{n}(x, \tau)\right)\right.} \\
&\left.+N\left(\sum_{n=0}^{\infty} p^{n} G_{n}(x, \tau)\right)\right] d \tau .
\end{aligned}
$$

Putting side by side expressions of identical powers of $p$ provides solutions of a variety of orders with the first term [15]

$$
G_{0}(x, t)=T(x, t) .
$$

\section{Complexity of the Homotopy Decomposition Method}

It is very important to test the computational complexity of a method or algorithm [15]. Complexity of an algorithm is the study of how long a program will take to run, depending on the size of its input and long of loops made inside the code [15]. We compute a numerical example which is solved by the homotopy decomposition method. The code has been presented with Mathematica 8 according to the following code [15].

Step 1. Set $m \leftarrow 0$.

Step 2. Calculate the recursive relation after the comparison of the terms of the same power is done.
Step 3. If $\left\|U_{n+1}(x, t)-U_{n}(x, t)\right\|<r$ with $r$ being the ratio of the neighbourhood of the exact solution [15], then go to Step 4 , else $m \leftarrow m+1$ and go to Step 2 .

Step 4. Print out

$$
G(x, t)=\sum_{n=0}^{\infty} U_{n}(x, t)
$$

as the approximate of the exact solution.

Lemma 1. If the exact solution of the fractional partial differential equation (4) exists, then

$$
\left\|G_{n+1}(x, t)-G_{n}(x, t)\right\|<r, \quad \forall(x, t) \in X \times T .
$$

Proof. Let $(x, t) \in X \times T$; since the exact solution exists, then we have the following:

$$
\begin{aligned}
\| G_{n+1} & (x, t)-G_{n}(x, t) \| \\
& =\left\|G_{n+1}(x, t)-G(x, t)+G(x, t)-G_{n}(x, t)\right\| \\
& \leq\left\|G_{n+1}(x, t)-G(x, t)\right\|+\left\|-G_{n}(x, t)+G(x, t)\right\| \\
& \leq \frac{r}{2}+\frac{r}{2}=r .
\end{aligned}
$$

The last inequality follows from [15].

Lemma 2. The complexity of the homotopy decomposition method is of order $O(n)$.

Proof. The number of computations including product, addition, subtraction, and division are the following.

In Step 2

$G_{0}: 0$ because it is obtained directly from the initial guess [15],

$$
\begin{aligned}
& G_{1}: 3, \\
& G_{n}: 3 .
\end{aligned}
$$

Now in Step 4 the total number of computations is equal to $\sum_{j=0}^{n} G_{j}(x, t)=3 n=O(n)$.

\section{Application}

In this section we apply this method for solving partial differential equation with time- and space-fractional derivatives.

Example 3. Let us consider the fractional Riccati differential equation

$$
D_{0}^{\alpha} y(x)=-y(x)^{2}+1, \quad 0<\alpha \leq 1, x>0
$$

subject to the initial condition

$$
y(0)=0
$$


Following the discussion presented in Section 3, we obtain the following:

$$
\begin{aligned}
& \sum_{n=0}^{\infty} p^{n} y_{n}(x) \\
& =y(0)-\frac{p}{\Gamma(\alpha)} \int_{0}^{x}(x-t)^{\alpha-1} \\
& \times\left[\left(\sum_{n=0}^{\infty} p^{n} y_{n}(t)\right)^{2}-1\right] d t .
\end{aligned}
$$

Comparing the terms of the same power of $p$ and using (18), we obtain the following integral equations:

$$
\begin{gathered}
p^{0}: y_{0}(x)=y(0)=0, \quad y_{0}(0)=0, \\
p^{1}: y_{1}(x)=-\frac{1}{\Gamma(\alpha)} \int_{0}^{x}(x-t)^{\alpha-1}\left[y_{0}^{2}(t)-1\right] d t \\
p^{n}: y_{n}(x)=-\frac{1}{\Gamma(\alpha)} \\
\times \int_{0}^{x}(x-t)^{\alpha-1} \sum_{j=0}^{n-1} y_{j} y_{n-j-1} d t \\
y_{n}(0)=0, n \geq 2 .
\end{gathered}
$$

And the following solutions are obtained:

$$
\begin{gathered}
y_{0}(x)=0, \\
y_{1}(x)=\frac{x^{\alpha}}{\Gamma(\alpha+1)}, \\
y_{2}(x)=0 \\
y_{3}(x)=-\frac{x^{3 \alpha} \Gamma(1+2 \alpha)}{\Gamma(1+3 \alpha) \Gamma^{2}(1+\alpha)}, \\
y_{4}(x)=0 \\
y_{5}(x)=\frac{2 x^{5 \alpha} \Gamma(1+2 \alpha) \Gamma(1+4 \alpha)}{\Gamma^{3}(1+\alpha) \Gamma(1+3 \alpha) \Gamma(1+5 \alpha)}, \\
y_{6}(x)=0, \\
y_{7}(x)=-\left(x^{7 \alpha} \Gamma(1+2 \alpha)\right. \\
\times(4 \Gamma(1+3 \alpha) \Gamma(1+4 \alpha) \\
\times\left(\Gamma^{3}(1+\alpha) \Gamma^{2}(1+3 \alpha) \Gamma(1+5 \alpha) \Gamma(1+7 \alpha)\right)^{-1},
\end{gathered}
$$

$$
\begin{gathered}
y_{8}(x)=0 \\
y_{9}(x)=\left(( x ^ { 9 \alpha } ) \left(\Gamma^{5}(1+\alpha) \Gamma^{3}(1+3 \alpha)\right.\right. \\
\left.\times \Gamma(1+5 \alpha) \Gamma(1+7 \alpha) \Gamma(1+9 \alpha))^{-1}\right) \\
\times\left(2 x^{9 \alpha} \Gamma(1+2 \alpha)\right. \\
\times(4 \Gamma(1+3 \alpha) \Gamma(1+4 \alpha) \Gamma(1+6 \alpha) \\
+\Gamma(1+2 \alpha)(\Gamma(1+4 \alpha) \Gamma(1+6 \alpha) \\
\times \Gamma(1+8 \alpha))
\end{gathered}
$$

Using the package Mathematica, in the same manner, one can obtain the rest of the components. But, here, 10 terms were computed and the asymptotic solution is given by

$$
y_{N=9}(x)=\sum_{n=0}^{9} y_{n}(x) \text {. }
$$

Now to access the accuracy of HDM, we compare the approximated solution (23) when $\alpha=1$ with the exact solution given as follows:

$$
y(x)=\tanh (x)
$$

Notice that when $\alpha=1$,

$$
\begin{aligned}
y_{N=9}(x) & =\sum_{n=0}^{9} y_{n}(x) \\
& =x-\frac{x^{3}}{3}+\frac{2 x^{5}}{15}-\frac{17 x^{7}}{315}+\frac{62 x^{9}}{2835} .
\end{aligned}
$$

Therefore, for any $N \geq 9$, we have

$$
y_{N}(x)=\sum_{n=0}^{N} \frac{2^{2 n}\left(2^{2 n}-1\right) x^{2 n-1}}{(2 n) !} B_{2 n}, \quad|x|<\frac{\pi}{2},
$$

with $B_{2 n}$ being the Bernoulli number. Now also notice that the Taylor series of $\tanh (x)$ of order 10 at the origin gives exactly $y_{N=9}(x)$. Thus, it is worth concluding that when $\alpha=1$ the approximated solution converges to the exact solution of Example 3.

Remark 4. Example 3 has been solved using HAM [24], ADM [25], VIM [26], and HPM [27, 28], and recently it was solved in [29]. Comparison of the results of applying the HDM with others methods leads to significant consequences.

An Eton proverb says "an image is equivalent to one thousand words." The following figure shows the comparison of the approximated solution and the exact solution. 


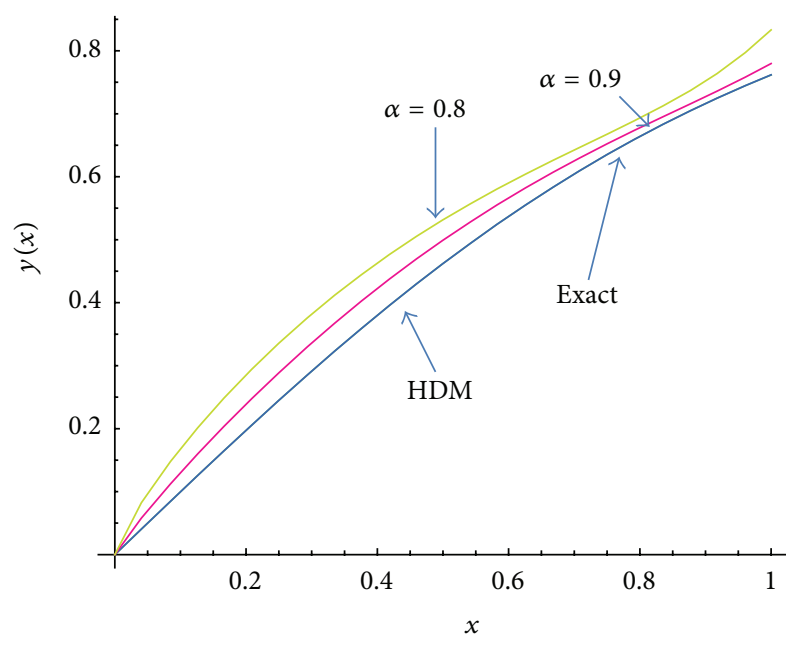

FIGURE 1: Comparison of approximated and exact solutions for $N=$ 15.

Figure 1 shows that the fractional solution decrease in the fractional order $\alpha$ causes an increase in the function. HDM solutions indicate that the present algorithm performs with extreme efficiency, simplicity, and reliability. The results obtained from HDM are fully compatible with the exact solution when $\alpha=1$, and are more accurate than those obtained from existing methods.

Example 5. Consider the following form of the timefractional equation:

$$
\begin{array}{r}
D_{t}^{\alpha} u=\frac{1}{2} u u_{x, x}-2 u^{2} u_{x}+u_{x}^{2}, \\
0<\alpha \leq 1, x>0,
\end{array}
$$

with the initial condition

$$
u(x, 0)=-\sqrt{c} \tanh (\sqrt{c} x)
$$

Following the steps of HDM, we obtain

$$
\begin{aligned}
\sum_{n=0}^{\infty} p^{n} u_{n}(x, t) & \\
= & u(x, 0)+\int_{0}^{y} \frac{1}{2}\left(\sum_{n=0}^{\infty} p^{n} u_{n}(x, y)\right)\left(\sum_{n=0}^{\infty} p^{n} u_{n}(x, y)\right)_{x, x} \\
& -2\left(\sum_{n=0}^{\infty} p^{n} u_{n}(x, y)\right)^{2}\left(\sum_{n=0}^{\infty} p^{n} u_{n}(x, y)\right)_{x} \\
& +\left(\left(\sum_{n=0}^{\infty} p^{n} u_{n}(x, y)\right)_{x} d y\right)^{2}
\end{aligned}
$$

Comparing the terms of the same power of $p$ and using (27), we obtain the following:

$$
\begin{aligned}
& p^{0}: u_{0}(x, t)= u(x, 0)=-\sqrt{c} \tanh (\sqrt{c} x), \\
& p^{1}: u_{1}(x, t)=\int_{0}^{t}\left(\frac{1}{2} u_{0}\left(u_{0}\right)_{x, x}-2\left(u_{0}\right)^{2}\left(u_{0}\right)_{x}\right. \\
&\left.+\left(\left(u_{0}\right)_{x}\right)^{2}\right) d \tau, \\
& p^{n}: u_{n}(x, t)=\int_{0}^{t} \frac{1}{2} \sum_{j=0}^{n-1} u_{j}\left(u_{n-1-j}\right)_{x, x}(x, 0)=0, \\
&-2 \sum_{j=0}^{n-1} \sum_{k=0}^{j} u_{j} u_{j-k}\left(u_{n-1-j}\right)_{x} \\
&+\sum_{j=0}^{n-1}\left(u_{j}\right)_{x}\left(u_{n-j-1}\right)_{x} d y, \\
& u_{n}(x, 0)=0, \quad n \geq 2 .
\end{aligned}
$$

The following solutions are obtained:

$$
\begin{aligned}
& u_{0}(x, t)=u(x, 0)=-\sqrt{c} \tanh (\sqrt{c} x), \\
& u_{1}(x, t)=\sqrt{c}\left(c^{3 / 2}-c^{3 / 2}(\tanh (\sqrt{c} x))^{2}\right) \frac{t^{\alpha}}{\Gamma(\alpha+1)}, \\
& u_{2}(x, t)=\sqrt{c}\left(\frac{1}{2} c^{3} \tanh (\sqrt{c} x)-\tanh (\sqrt{c} x)\right. \\
& \left.\times\left(-\frac{c^{3}}{2}+c^{3}(\tanh (\sqrt{c} x))^{2}\right)\right) t^{2 \alpha} \\
& u_{3}(x, t)=\sqrt{c}\left(\frac{\sqrt[9]{c}}{6}-\frac{1}{2} \sqrt[9]{c}(\tanh (\sqrt{c} x))^{2}\right. \\
& +\sqrt[3]{c}\left(-\frac{c^{3}}{2}+c^{3}(\tanh (\sqrt{c} x))^{2}\right) \\
& -\tanh (\sqrt{c} x) \\
& \times\left(-\frac{1}{3} \sqrt[9]{c} \tanh (\sqrt{c} x)+\sqrt[3]{c} \tanh (\sqrt{c} x)\right. \\
& \left.\left.\times\left(-\frac{c^{3}}{2}+c^{3}(\tanh (\sqrt{c} x))^{2}\right)\right)\right) t^{3 \alpha},
\end{aligned}
$$




$$
\begin{aligned}
& u_{4}(x, t) \\
& =\sqrt{c}\left(\frac{1}{8} c^{6} \tanh (\sqrt{c} x)-\frac{1}{2} c^{3} \tanh (\sqrt{c} x)\right. \\
& \times\left(-\frac{c^{3}}{2}+c^{3}(\tanh (\sqrt{c} x))^{2}\right) \\
& +\sqrt[3]{c}\left(-\frac{1}{3} \sqrt[9]{c} \tanh (\sqrt{c} x)+\sqrt[3]{c} \tanh (\sqrt{c} x)\right. \\
& \left.\times\left(-\frac{c^{3}}{2}+c^{3}(\tanh (\sqrt{c} x))^{2}\right)\right)-\tanh (\sqrt{c} x) \\
& \quad+\frac{c^{6}(\tanh (\sqrt{c} x))^{2}}{6} \\
& \times\left(\frac{\sqrt[9]{c} c^{3}\left(-\frac{c^{3}}{2}+c^{3}(\tanh (\sqrt{c} x))^{2}\right)}{3} \sqrt[3]{c} \tanh (\sqrt{c} x)\right. \\
& \left.\left.\quad\left(-\frac{c^{3}}{2}+c^{3}(\tanh (\sqrt{c} x))^{2}\right)\right)\right) t^{4 \alpha} .
\end{aligned}
$$

Using the package Mathematica, in the same manner, one can obtain the rest of the components. But in this case, 5 terms were computed and the asymptotic solution is given by

$$
\begin{aligned}
u_{N=5}(x, t)= & u_{0}(x, t)+u_{1}(x, t) \\
& +u_{2}(x, t)+u_{3}(x, t)+u_{4}(x, t)+\cdots .
\end{aligned}
$$

Now notice that if we set $\alpha=1$, we recover the Taylor series of $\sqrt{c} \tanh (\sqrt{c}(x-c t))$ of order 4 . Therefore, if $\alpha=1$ using the package Mathematica, we recover

$$
\sum_{n=0}^{\infty} u_{n}(x, t)=-\sqrt{c} \tanh (\sqrt{c}(x-c t)) .
$$

This is the exact solution of Example 5.

In this section, to access the accuracy of the HDM, we compare the approximation (32) with the exact solution; this is depicted in Figures 2 and 3.

From the Figure 2, it is obvious that when $\alpha=1$, the solution is nearly identical with the exact solution.

Example 6. Consider the fractional predator-prey equation

$$
\begin{array}{r}
\frac{\partial^{\alpha} u}{\partial t^{\alpha}}=\frac{\partial^{2} u^{2}}{\partial x^{2}}+\frac{\partial^{2} u^{2}}{\partial y^{2}}+a u-b u v, \\
u(x, y, 0)=e^{x+y},
\end{array}
$$

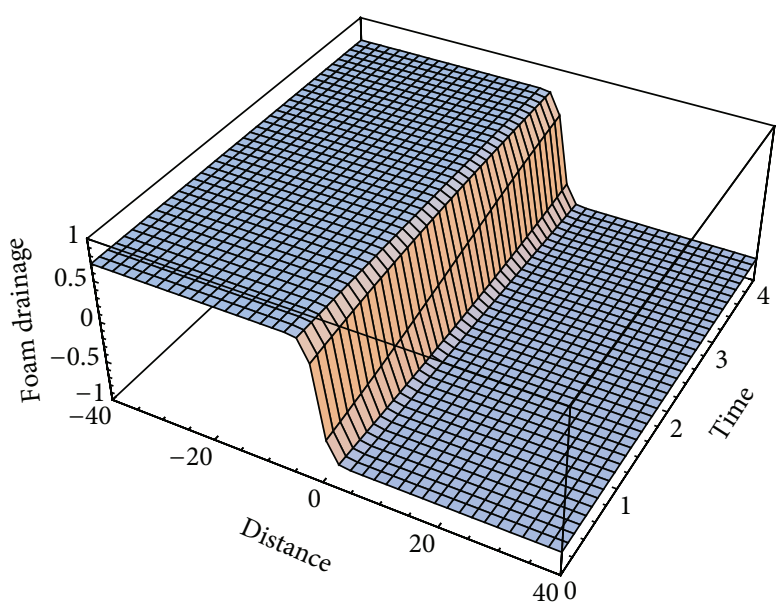

FIGURE 2: Exact solution for $c=1 / 2$.

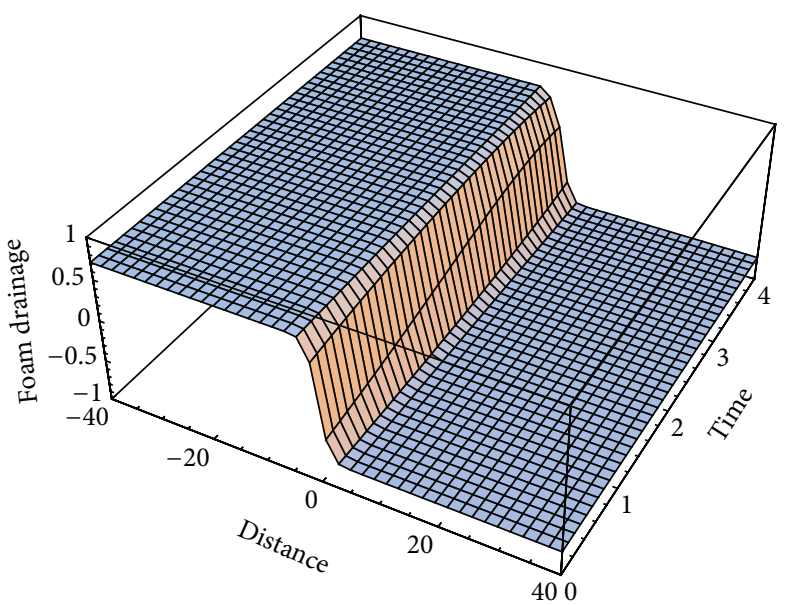

FIGURE 3: Approximated solution for $c=1 / 2$ and $N=6$.

$$
\begin{array}{r}
\frac{\partial^{\beta} v}{\partial t^{\beta}}=\frac{\partial^{2} v^{2}}{\partial x^{2}}+\frac{\partial^{2} v^{2}}{\partial y^{2}}+b v u-c v, \\
v(x, y, 0)=e^{x+y} .
\end{array}
$$

Following the homotopy decomposition method, presented in Section 3, we obtain the following system:

$$
\begin{aligned}
\sum_{n=0}^{\infty} p^{n} u_{n}= & u(x, y, 0) \\
& +\frac{p}{\Gamma(\alpha)} \int_{0}^{t}\left(\partial_{x x}\left(\sum_{n=0}^{\infty} p^{n} u_{n}\right)^{2}+\partial_{y y}\left(\sum_{n=0}^{\infty} p^{n} u_{n}\right)^{2}\right. \\
& \left.+a \sum_{n=0}^{\infty} p^{n} u_{n}-b \sum_{n=0}^{\infty} p^{n} u_{n} \sum_{n=0}^{\infty} p^{n} v_{n}\right) \\
& \times(t-\tau) d \tau,
\end{aligned}
$$




$$
\begin{aligned}
\sum_{n=0}^{\infty} p^{n} v_{n}=v(x, y, 0) & +\frac{p}{\Gamma(\beta)} \\
\times & \int_{0}^{t}\left(\partial_{x x}\left(\sum_{n=0}^{\infty} p^{n} v_{n}\right)^{2}+\partial_{y y}\left(\sum_{n=0}^{\infty} p^{n} v_{n}\right)^{2}\right. \\
+ & b \sum_{n=0}^{\infty} p^{n} u_{n} \sum_{n=0}^{\infty} p^{n} v_{n} \\
& \left.-c \sum_{n=0}^{\infty} p^{n} u_{n}\right)(t-\tau)^{\beta-1} d \tau
\end{aligned}
$$

Comparing the terms of the same power of $p$ yields the following two sets of linear equation:

$$
\begin{aligned}
& p^{0}: u_{0}=u(x, y, 0)=e^{x+y}, \\
& p^{1}: u_{1}=\frac{1}{\Gamma(\alpha)} \int_{0}^{t}(t-\tau)^{\alpha-1} \\
& \times\left(\partial_{x x} u_{0}^{2}+\partial_{y, y} u_{0}^{2}+a u_{0}-b u_{0} v_{0}\right) d \tau, \\
& u_{1}(x, y, 0)=0 \text {, } \\
& p^{n}: u_{n}(x, y, t) \\
& =\frac{1}{\Gamma(\alpha)} \int_{0}^{t}(t-\tau)^{\alpha-1} \\
& \times\left(\sum_{j=0}^{n-1}\left(u_{j}\right)_{x x}\left(u_{n-j-1}\right)_{x x}\right. \\
& +\sum_{j=0}^{n-1}\left(u_{j}\right)_{y, y}\left(u_{n-j-1}\right)_{y y} \\
& \left.+a u_{n-1}-b \sum_{j=0}^{n-1} u_{j} v_{n-j-1}\right) d \tau, \\
& u_{n}(x, y, 0)=0, \quad n \geq 2, \\
& p^{0}: v_{0}=v(x, y, 0)=e^{x+y} \text {, } \\
& p^{1}: v_{1}=\frac{1}{\Gamma(\beta)} \int_{0}^{t}(t-\tau)^{\alpha-1} \\
& \times\left(\partial_{x, x} v_{0}^{2}+\partial_{y, y} v_{0}^{2}+b u_{0} v_{0}-c v_{0}\right) d \tau, \\
& u_{1}(x, y, 0)=0 \text {, } \\
& p^{n}: v_{n}(x, y, t) \\
& =\frac{1}{\Gamma(\beta)} \int_{0}^{t}\left(\sum_{j=0}^{n-1}\left(v_{j}\right)_{x x}\left(v_{n-j-1}\right)_{x x}\right. \\
& +\sum_{j=0}^{n-1}\left(v_{j}\right)_{y, y}\left(v_{n-j-1}\right)_{y y}
\end{aligned}
$$

$$
\begin{gathered}
\left.+b \sum_{j=0}^{n-1} u_{j} v_{n-j-1}-c v_{n-1}\right), \\
\times(t-\tau)^{\beta-1} d \tau, \quad v_{n}(x, y, 0)=0, \quad n \geq 2 .
\end{gathered}
$$

The following solutions are obtained:

$$
\begin{aligned}
& u_{0}=u(x, y, 0)=e^{x+y}, \\
& v_{0}=v(x, y, 0)=e^{x+y}, \\
& u_{1}=\frac{e^{x+y}\left(a-\left(-8+b e^{x+y}\right)\right) t^{\alpha}}{\Gamma(1+\alpha)}, \\
& v_{1}=\frac{e^{x+y}\left(-c+(8+b) e^{x+y}\right) t^{\beta}}{\Gamma(1+\beta)}, \\
& u_{2}=\frac{1}{\Gamma(1+\alpha) \Gamma(1+\beta) \Gamma((1 / 2)+\beta) \Gamma(1+\alpha+\beta)} \\
& \times\left(4^{-\beta} e^{x+y} t^{\beta}\right. \\
& \times\left(b e^{x+y}\left(4^{\beta} a+\left(2^{3+2 \beta}-4^{\beta} b\right) e^{x+y}\right)\right. \\
& \times t^{\alpha} \Gamma(1+\beta) \Gamma(1+\alpha) \Gamma\left(\frac{1}{2}+\beta\right) \\
& +\left(c^{2}-2(6+b) c e^{x+y}\right) \\
& +\left(128+24 b+b^{2}\right) \sqrt{\pi} t^{\beta} \Gamma(1+\alpha) \\
& \left.\left.\times \Gamma\left(\frac{1}{2}+\beta\right)\right)\right), \\
& u_{2}=\frac{1}{\Gamma(1+\alpha) \Gamma(1+\beta) \Gamma((1 / 2)+\beta) \Gamma(1+\alpha+\beta)} \\
& \times\left(4^{-\alpha} e^{x+y} t^{\alpha}\right. \\
& \times\left(-b e^{x+y}\left(-4^{\alpha} c+\left(2^{3+2 \beta}+4^{\alpha} b\right) e^{x+y}\right)\right. \\
& \times t^{\beta} \Gamma(1+\beta) \Gamma(1+\alpha) \Gamma\left(\frac{1}{2}+\beta\right) \\
& +\left(c^{2}-2(-6+b) a e^{x+y}\right) \\
& +\left(128-24 b+b^{2}\right) \sqrt{\pi} t^{\alpha} \Gamma(1+\alpha) \\
& \left.\left.\times \Gamma\left(\frac{1}{2}+\beta\right)\right)\right) .
\end{aligned}
$$


TABLE 1: Comparison of the numerical results of approximate solutions obtained via HDM, VIM and HPM with exact solution of (27).

\begin{tabular}{lcccccc}
\hline Variable $x$ & HDM & VIM & HPM & Exact & $E 1=|H D M-E X|$ & $E 2=\mid$ VIM - EX $\mid$ \\
\hline 1.05 & 0.78162 & 0.781619 & 0.78162 & 0.781806 & 0.000186617 & 0.000187358 \\
1.06 & 0.785437 & 0.785426 & 0.785437 & 0.785664 & 0.000226381 & 0.000237859 \\
1.07 & 0.789187 & 0.789179 & 0.789187 & 0.789461 & 0.000274099 & 0.000282221 \\
1.08 & 0.792868 & 0.792789 & 0.792868 & 0.793199 & 0.000331261 & 0.000410097 \\
1.09 & 0.796479 & 0.796467 & 0.796479 & 0.796878 & 0.000399615 & 0.000411144 \\
1.10 & 0.800018 & 0.810017 & 0.800018 & 0.800499 & 0.000481214 & 0.00951798 \\
1.11 & 0.803484 & 0.8100484 & 0.804062 & 0.804062 & 0.000578458 & 0.00598601 \\
\hline
\end{tabular}

The numerical results show that the HPM and HDM are more accurate than the VIM in this case.

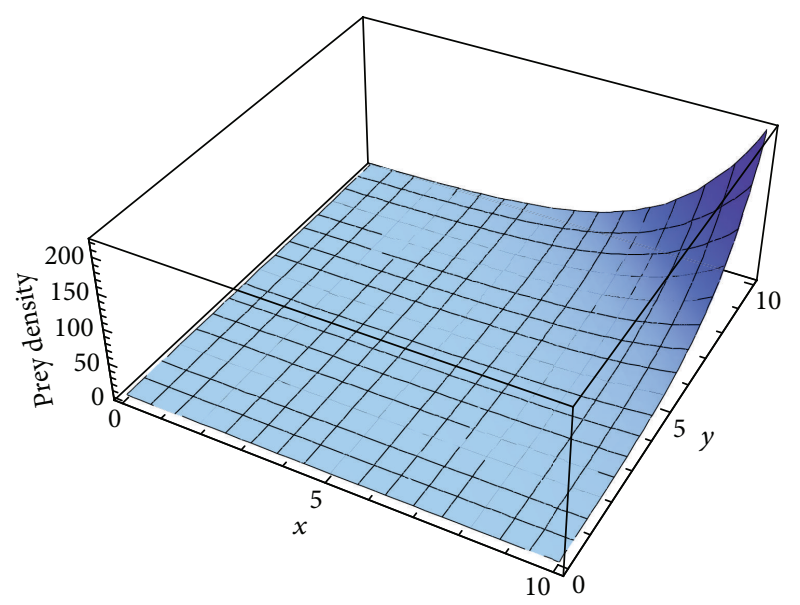

Figure 4: Prey density for $t=0.053, \alpha=0.88, \beta=0.542, a=$ 0.5 , and $c=0.3$.

Using the package Mathematica, in the same manner, one can obtain the rest of the components. But in this case, 3 terms were computed and the asymptotic solution is given by

$$
\begin{aligned}
& u_{N=3}(x, t)=u_{0}(x, t)+u_{1}(x, t)+u_{2}(x, t)+\cdots \\
& v_{N=3}(x, t)=v_{0}(x, t)+v_{1}(x, t)+v_{2}(x, t)+\cdots
\end{aligned}
$$

Figures 4 and 5 show the numerical solutions for preypredator population system with appropriate parameter. From the figures, we recognize that prey population density first increases with the spatial variables but will increase less than the predator population. However, the predator population density always increases with the spatial variables with the parameter we choose here. Analysis and results of prey-predator population system indicate that the fractional model matches the regular biological diffusion behavior observed in the field. From the figures, it is also clear to see the time evolution of prey-predator population density and we also know that the numerical solutions of fractional prey-predator population model are continuous with the parameters $\alpha$ and $\beta$.

To test the accuracy of the method used in this paper, we present the numerical result of the approximate solution of (27) and the numerical results of the exact solution in Table 1.

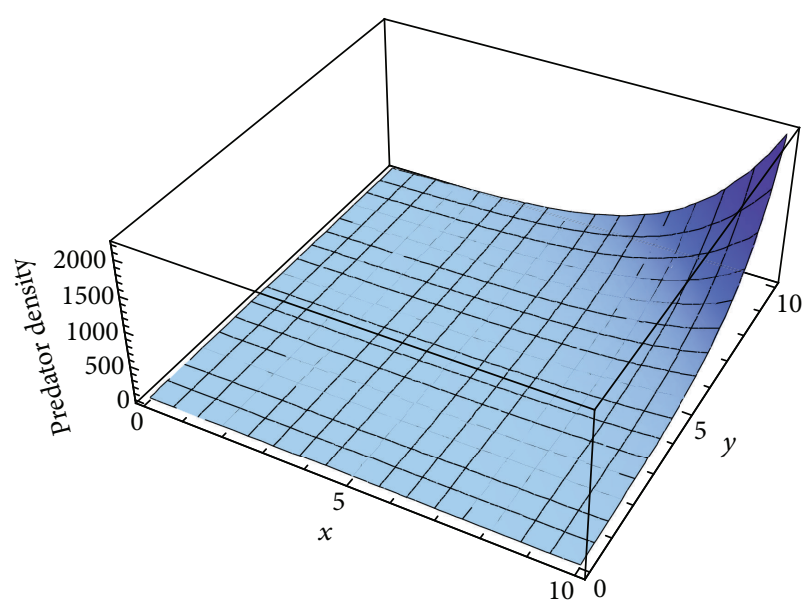

Figure 5: Predator density for $t=0.053, \alpha=0.88, \beta=0.542$, $a=0.5$, and $c=0.3$.

The numerical results show that the HPM and HDM are more accurate than the VIM in this case.

\section{Conclusion}

In this paper, we have productively developed HDM for solving partial differential equation of space- and timefractional derivatives. the dependability of the HDM and its wider applicability to fractional differential equation. It is consequently, that the HDM makes available more practical series solutions that converge very speedily in real physical problems. Also, the amount of the computational effort has been abridged. In the bargain, the computations concerned in HDM are very straightforward. It is established that HDM is a prevailing and resourceful instrument for FPDEs.

\section{Conflict of Interests}

The authors declare that there is no conflict of interests for this paper.

\section{Authors' Contribution}

The first draft of this paper was portrayed by Abdon Atangana; the revised version was carefully corrected by Samir 
Brahim Belhaouari, and both authors read and submitted the final version.

\section{Acknowledgment}

The authors will like to thank the reviewer for his valuable spare time to read this paper and for his valuable comments and suggestions toward the enhancement of this paper.

\section{References}

[1] L. Blank, "Numerical treatment of differential equations of fractional order," Nonlinear World, vol. 4, no. 4, pp. 473-491, 1997.

[2] M. Caputo, "Linear models of dissipation whose Q is almost frequency independent," Journal of the Royal Australian Historical Society, vol. 13, pp. 529-539, 1967.

[3] K. B. Oldham and J. Spanier, The Fractional Calculus, Academic Press, New York, NY, USA, 1974.

[4] S. Momani and N. Shawagfeh, "Decomposition method for solving fractional Riccati differential equations," Applied Mathematics and Computation, vol. 182, no. 2, pp. 1083-1092, 2006.

[5] Z. Odibat and S. Momani, "Modified homotopy perturbation method: application to quadratic Riccati differential equation of fractional order," Chaos, Solitons \& Fractals, vol. 36, no. 1, pp. 167-174, 2008.

[6] Z. Odibat and S. Momani, "Application of variation iteration method to nonlinear differential equations of fractional order," International Journal of Nonlinear Sciences and Numerical Simulation, vol. 1, no. 7, pp. 15-27, 2006.

[7] S. J. Liao, The proposed homotopy analysis technique for the solution of nonlinear problems [Ph.D. thesis], Jiao Tong University, 1992.

[8] S. Liao, Beyond Perturbation: Introduction to the Homotopy Analysis Method, vol. 2 of CRC Series: Modern Mechanics and Mathematics, Chapman \& Hall/CRC, Boca Raton, Fla, USA, 2003.

[9] A. S. Bataineh, A. K. Alomari, M. S. M. Noorani, I. Hashim, and R. Nazar, "Series solutions of systems of nonlinear fractional differential equations," Acta Applicandae Mathematicae, vol. 105, no. 2, pp. 189-198, 2009.

[10] M. Dehghan, J. Manafian, and A. Saadatmandi, "Solving nonlinear fractional partial differential equations using the homotopy analysis method," Numerical Methods for Partial Differential Equations, vol. 26, no. 2, pp. 448-479, 2010.

[11] W. Chen, L. Ye, and H. Sun, "Fractional diffusion equations by the Kansa method," Computers \& Mathematics with Applications, vol. 59, no. 5, pp. 1614-1620, 2010.

[12] Z.-J. Fu, W. Chen, and H.-T. Yang, "Boundary particle method for Laplace transformed time fractional diffusion equations," Journal of Computational Physics, vol. 235, pp. 52-66, 2013.

[13] Q. Liu, Y. T. Gu, P. Zhuang, F. Liu, and Y. F. Nie, "An implicit RBF meshless approach for time fractional diffusion equations," Computational Mechanics, vol. 48, no. 1, pp. 1-12, 2011.

[14] A. Atangana and J. F. Botha, "Analytical solution of groundwater flow equation via Homotopy Decomposition Method," Journal of Earth Science \& Climatic Change, vol. 3, p. 115, 2012.

[15] A. Atangana and A. Secer, "The time-fractional coupled-Korteweg-de-Vries equations," Abstract and Applied Analysis, vol. 2013, Article ID 947986, 8 pages, 2013.

[16] A. Atangana, "New class of boundary value problems," Information Sciences Letters, vol. 1, no. 2, pp. 67-76, 2012.
[17] J.-H. He, "Homotopy perturbation technique," Computer Methods in Applied Mechanics and Engineering, vol. 178, no. 3-4, pp. 257-262, 1999.

[18] K. S. Miller and B. Ross, An Introduction to the Fractional Calculus and Fractional Differential Equations, John Wiley \& Sons, New York, NY, USA, 1993.

[19] I. Podlubny, Fractional Differential Equations, vol. 198 of Mathematics in Science and Engineering, Academic Press, San Diego, Calif, USA, 1999.

[20] S. G. Samko, A. A. Kilbas, and O. I. Marichev, Fractional Integrals and Derivatives, Gordon and Breach Science Publishers, Yverdon, Switzerland, 1993.

[21] G. Jumarie, "On the representation of fractional Brownian motion as an integral with respect to $(\mathrm{d} t)^{a}$, , Applied Mathematics Letters, vol. 18, no. 7, pp. 739-748, 2005.

[22] G. Jumarie, "Modified Riemann-Liouville derivative and fractional Taylor series of nondifferentiable functions further results," Computers \& Mathematics with Applications, vol. 51, no. 9-10, pp. 1367-1376, 2006.

[23] H. H. Fadravi, H. S. Nik, and R. Buzhabadi, "Homotopy analysis method for solving foam drainage equation with space- and time-fractional derivatives," International Journal of Differential Equations, vol. 2011, Article ID 237045, 12 pages, 2011.

[24] Y. Tan and S. Abbasbandy, "Homotopy analysis method for quadratic Riccati differential equation," Communications in Nonlinear Science and Numerical Simulation, vol. 13, no. 3, pp. 539-546, 2008.

[25] S. Momani and N. Shawagfeh, "Decomposition method for solving fractional Riccati differential equations," Applied Mathematics and Computation, vol. 182, no. 2, pp. 1083-1092, 2006.

[26] M. Merdan, "On the solutions fractional Riccati differential equation with modified Riemann-Liouville derivative," International Journal of Differential Equations, vol. 2012, Article ID 346089, 17 pages, 2012.

[27] J. F. Botha and A. H. Cloot, "A generalized groundwater flow equation using the concept of non-integerorder," Water $S A$, vol. 32, no. 1, 2006.

[28] J. Cang, Y. Tan, H. Xu, and S.-J. Liao, "Series solutions of non-linear Riccati differential equations with fractional order," Chaos, Solitons and Fractals, vol. 40, no. 1, pp. 1-9, 2009.

[29] A.-M. Wazwaz, "A reliable modification of Adomian decomposition method," Applied Mathematics and Computation, vol. 102, no. 1, pp. 77-86, 1999. 


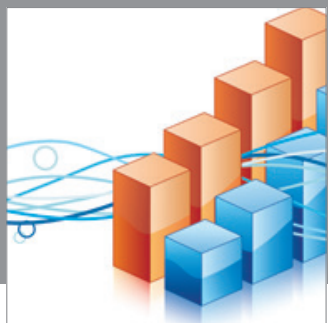

Advances in

Operations Research

mansans

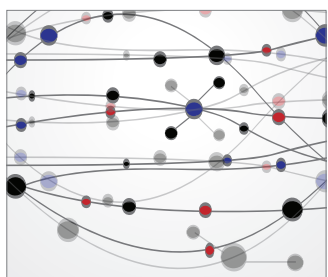

The Scientific World Journal
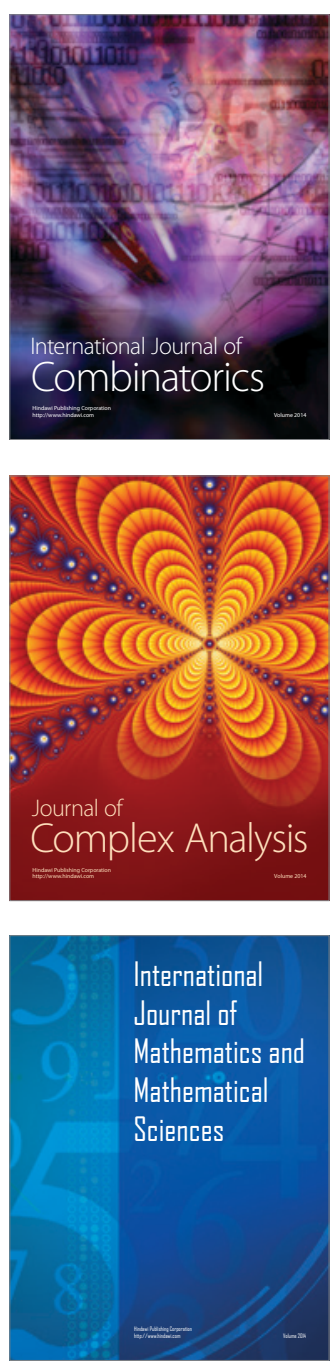
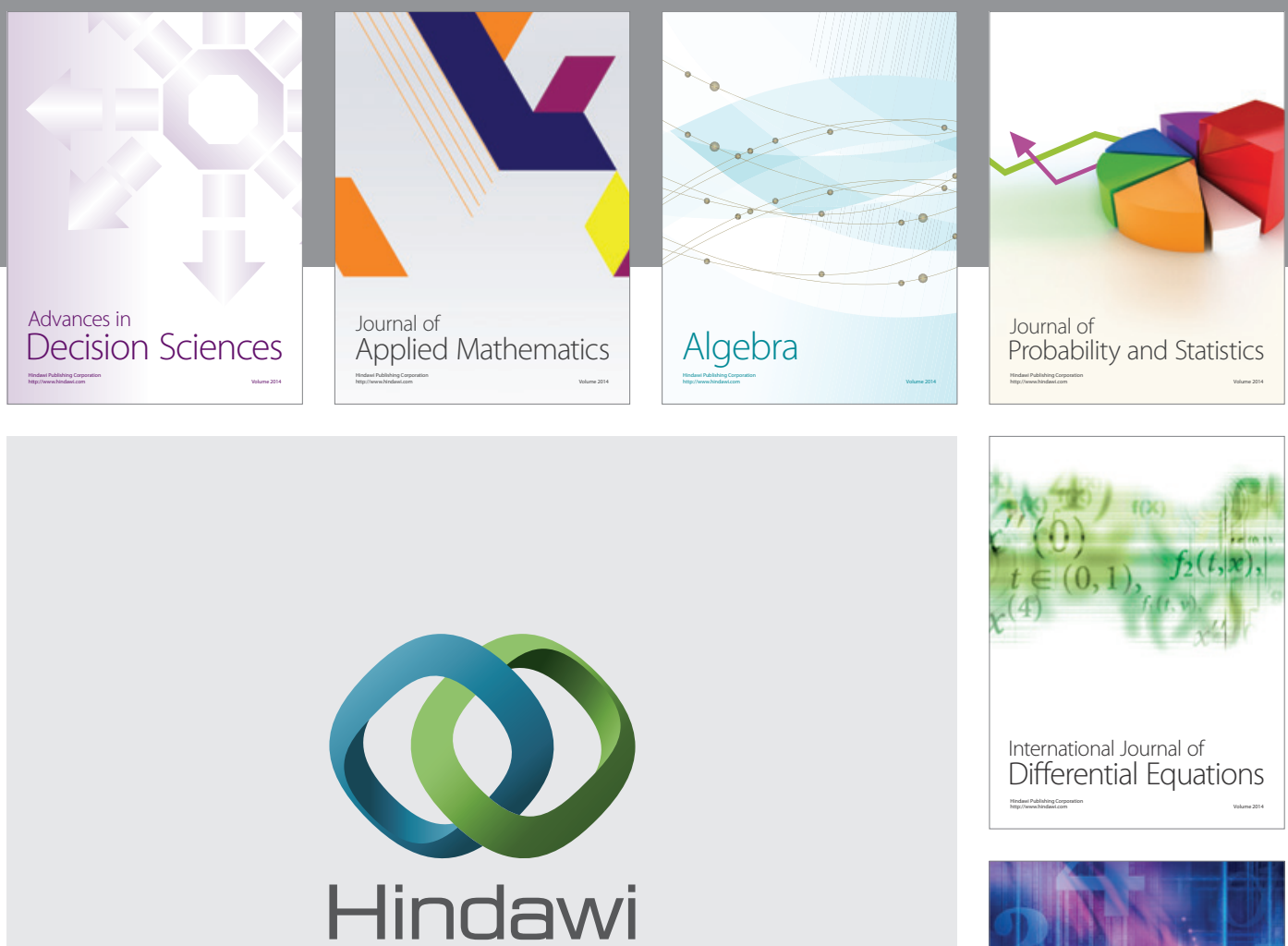

Submit your manuscripts at http://www.hindawi.com
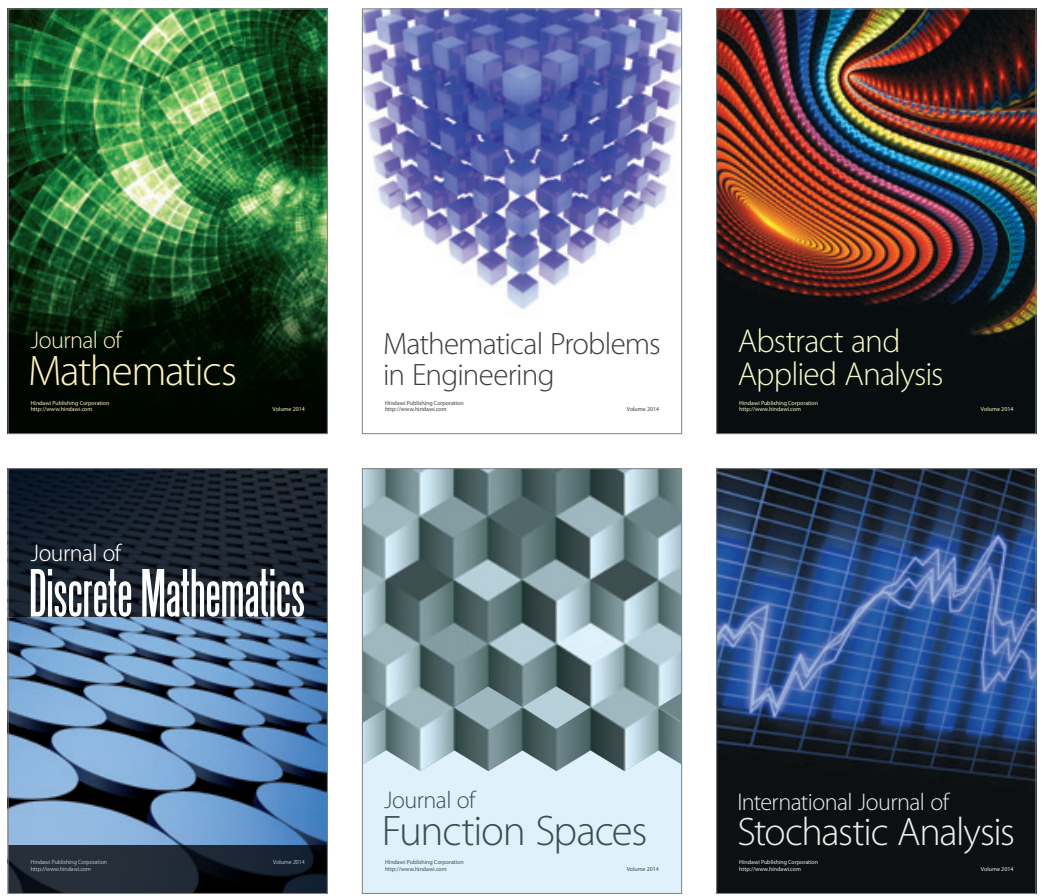

Journal of

Function Spaces

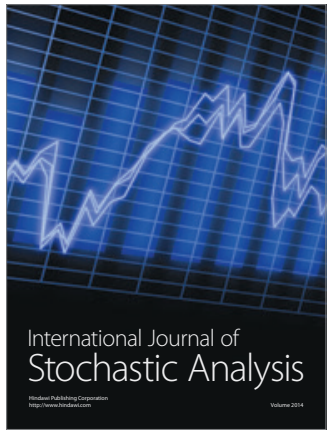

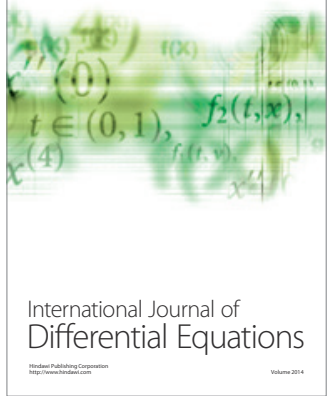
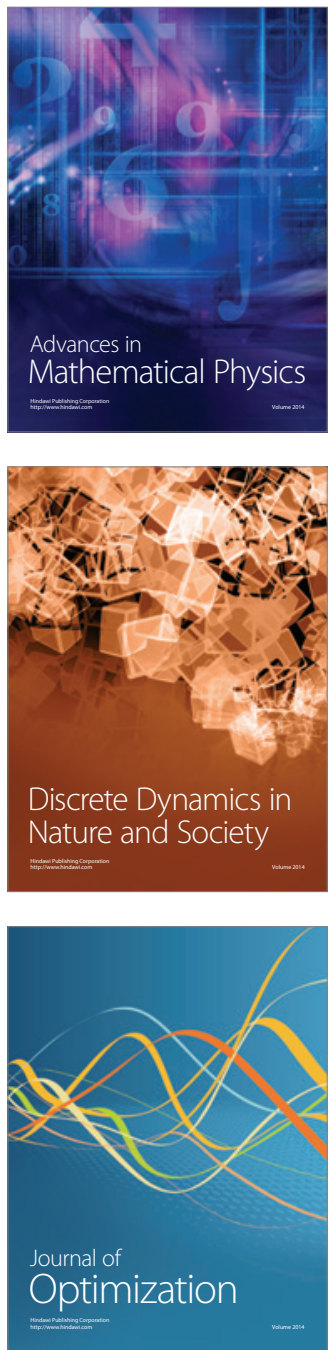